
Application to home care compared to hospital care

Raphaël Remonnay, Nathalie Havet, Magali Morelle, Marie-Odile Carrère 


\section{ANALYZING THE DETERMINANTS OF WILLINGNESS-TO- PAY VALUES FOR TESTING THE VALIDITY OF THE CONTINGENT VALUATION METHOD. APPLICATION TO HOME CARE COMPARED TO HOSPITAL CARE}

RAPHAEL REMONNAY, NATHALIE HAVET $\uparrow$, MAGALI MORELLE, MARIE-ODILE CARRERE

University of Lyon, Lyon, F-69003, France; CNRS, UMR 5824, GATE, Ecully, F-69130, France; ENS LSH, Lyon, F-69007, France ; Centre Leon Berard, Lyon, F-69003, France

${ }^{\dagger}$ Assistant Professor.

${ }^{\ddagger}$ Professor.

Running head: Testing contingent valuation validity

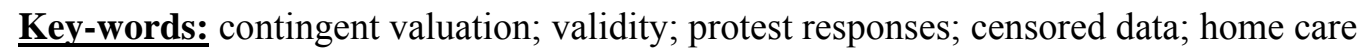

Name, address, telephone number and email address of the corresponding author:

Raphaël Remonnay

Axe Economie de la Santé, GATE, UMR 5824 CNRS,

Centre Léon Bérard, 28 rue Laennec, 69008 Lyon, France

Tel: (33).4.78.78.27.80 Fax: (33).4.78.78.28.04 E-mail: remonnay@lyon.fnclcc.fr

Word count (text ):5347 Figures: $0 \quad$ Tables: 3

Financial support: Our study received financial support from the French Ministry of Health. 


\section{SUMMARY}

The contingent valuation $(\mathrm{CV})$ method is an attractive approach for comparing home care to hospital care in which the only difference is patients' well-being during the treatment process and not health outcomes. We considered the empirical situation of blood transfusion (BT) in cancer patients and collected willingness to pay (WTP) values among BT users.

Our main objective was to test the validity of the CV method, namely its ability to elicit true preferences. Firstly, possible determinants of WTP values and their expected influences were identified, from both economic and non economic literature and from the findings of a pilot study.

Secondly, they were compared to predicted influences resulting from appropriate econometric analysis of WTP values elicited by a bidding process. From the health economics literature it appeared that the double-hurdle model is the most appropriate approach to account for zero values and protest responses. However, because the number of protest responses was too small, we used a truncated regression model.

None of the 7 hypothesized influences was invalidated by econometric results. The anchoring bias hypothesis was confirmed. The WTP for home BT compared to hospital BT increased with household income, with previous experience of home care, with living far from the hospital and with low quality of life. Conversely, it was lower for advanced-stage (palliative or terminal) than for early-stage (curative) patients.

We conclude that the CV approach is acceptable to severely ill patients. Moreover, WTP values demonstrate good validity given that influences predicted by our model are consistent with expected determinants. 


\section{INTRODUCTION}

In recent years, a growing number of studies conducted in the health care field have used contingent valuation (CV), as reported in several literature reviews (Diener et al., 1998; Olsen and Smith, 2001; Smith, 2003; Yeung et al., 2006). The CV method has been used for evaluating all types of health care strategies, either preventive, diagnostic or therapeutic. Meanwhile, its ability to provide the "true" preferences of the respondents has been extensively questioned. Accordingly, numerous validity properties such as criterion validity, content validity and construct validity have been defined and empirically tested using willingness-to-pay (WTP) responses.

Analyzing the determinants of WTP values can be a valuable way of assessing the validity of the $\mathrm{CV}$ method. Indeed, possible determinants and their expected influences can be derived from theoretical predictions or empirical literature, then confirmed or not using WTP data. For example, the respondent income should positively influence WTP values according to construct validity (Donaldson, 1999; Smith et al., 1999 ; Drummond et al., 2005). However, analyzing the determinants of WTP has received limited attention in health economics. The empirical literature about WTP has focused more on the method used for data collection and on possible consequences on the monetary values obtained (Smith 2003; Smith 2006; Smith $\left.2007^{\mathrm{a}, \mathrm{b}}\right)$

The technique used for econometric analysis of WTP data is nevertheless an important issue because an inappropriate choice can lead to erroneous inferences about the determinants of WTP and consequently about validity. Econometric modeling should fit the type of WTP question asked (Donaldson et al., 1995; Donaldson et al., 1998; Dalmau-Matarrodona, 2001; 
Kurth et al., 2004). A closed-ended approach and a dichotomous choice with follow-up provide qualitative binary responses while a payment scale approach, an open-ended question and a bidding process generate continuous monetary WTP values (Donaldson et al., 1998). In the 3 latter cases, the WTP distribution is usually censored so that the data may contain a large proportion of zero values, for which there is a range of possible explanations such as protest responses or real zeros consistent with economic decisions.

The objective of the present study was to analyze the determinants of WTP values in the framework of a CV survey aimed at comparing hospital care to home care. Home care is a promising alternative to hospital care in many health care situations. However, to our knowledge, the two options have never been compared using the CV approach.

We shall first present the empirical situation studied, the CV study design, the WTP questioning process, the selection of possible determinants of WTP values and sample characteristics. Secondly, the health economics literature on the econometric methods currently used for analyzing determinants of WTP data will be investigated, with a special focus on censored data and protest responses. Thirdly, the WTP values obtained will be analyzed using appropriate econometric modeling, and results will be compared to the expected influences of pre selected possible determinants. In conclusion, we will discuss the implications of our study regarding the validity of the CV method. 


\section{EMPIRICAL SITUATION, CV STUDY DESIGN AND DATA COLLECTION}

\subsection{Empirical situation}

$\mathrm{CV}$ is an attractive alternative to the Quality-adjusted life years (QALYs) approach when it comes to comparing two types of health care management which differ only in patients' wellbeing during the treatment process and not in health outcomes (Ryan and Shackley, 1995; Olsen and Smith, 2001). This is the case for some treatments which can be delivered at home or in the hospital day-care unit, such as blood transfusion (BT) in cancer care. BT is currently administered to advanced-stage cancer patients for curative, palliative or terminal intent. BT is simple to administer, with identical effectiveness and safety at home, in the framework of a homecare network, or in the hospital (Idri et al., 1996; Madgwick and Yardumian, 1999). Administration at home requires neither transportation between home and hospital nor waiting time in the hospital and may decrease the patient's tiredness; however, it may also increase the feeling of insecurity and isolation. As a result, home administration compared to hospital administration may be viewed differently from one patient to another.

\subsection{Study design}

A CV survey conducted in the Comprehensive Cancer Centre of the Rhone-Alps Region in Lyon, France, compared hospital BT to home BT. As said before, our analysis focused on WTP values. Willingness-to-accept (WTA) values were also collected for further analysis.

A prospective pilot study using the same design as the main study was conducted in 40 eligible patients to assess whether a CV survey was feasible in this situation, i.e. among patients personally involved in the treatment under consideration, most of them severely ill. 
We also aimed at identifying possible determinants of $\mathrm{CV}$ responses specific to the empirical situation under investigation.

The pilot study demonstrated the feasibility of the CV survey: of the 44 patients planned, only 4 did not consent to participate because they felt too tired for the 45 -minute interview. Therefore, a prospective study was conducted on a 12 months period in 2003 and 2004. All cancer patients needing a BT and aged more than 18 years were asked to participate. All were eligible to receive BT either at home or in the hospital. They were allocated between home BT and hospital BT according to organizational possibilities. For example, home BT was only available in a few well defined geographical areas. After obtaining informed consent, and less than 48 hours after BT administration, face-to-face interviews were conducted by a trained interviewer on the basis of a detailed guide. Although costly, this type of interview was chosen for minimizing hypothetical biases and improving the quality and rate of responses (Mitchell and Carson, 1989; NOAA, 1993, 1994; Smith et al., 1999a).

Patients were first asked whether they had already undergone home BT (including the current procedure when it was administered at home) and whether they had already received home care, other than BT. They were then given a detailed presentation of the two BT managements, either at home or in the hospital, and were told that effectiveness and safety were identical in both cases. Finally, they were asked where they would choose to receive their next BT, would they need another one in the future and would the two BT managements be available. 


\subsection{WTP questioning process}

Once they have chosen the BT arrangements, all patients were given a general presentation of the CV method, which was introduced as a way of measuring their preference for the type of BT management they had chosen, as compared to the other one, without consideration of the corresponding costs for the health care system. Then they were asked to imagine a hypothetical future situation where they would need another BT and where the only freely available management would be the one they had not chosen. Then the interviewer asked them how much they would be willing to pay to get their preferred BT management rather than the free alternative. We chose out-of-pocket expenditure which is the recommended payment vehicle when respondents are personally involved (Smith, 2003; Mitchell and Carson, 1989; O'Brien and Gafni, 1996). Thus, in reference to prospect theory (Kahneman and Tversky, 1979), WTP was framed as a gain (Sayman and Öncüller, 2005). Indeed, we considered that framing the WTP question as a gain rather than a loss would be easier to understand.

For asking WTP questions we preferred using a bidding process rather than closed-ended questions because of our small sample size (Donaldson et al., 1998). The process involved three steps. First, an initial bid of money was proposed to the patients. If they agreed to pay, we proposed a higher bid, whereas if they did not we proposed a lower one. This step was repeated twice. Patients were randomly assigned to two initial bids of money ( $38 €$ and $76 €$ ) to test for a possible anchoring bias affecting content validity (Herriges and Shogren, 1994; Flachaire and Hollard, 2007). The range from $38 €$ to $76 €$ was chosen because it was close to the range of fees normally covered by social insurance in France for a home visit by a specialist (including call-out charges). 


\subsection{Selection of possible determinants of WTP values}

As said before, a possible anchoring bias was tested: according to health economics literature, choosing a higher initial bid value could increase WTP responses, but would in no way decrease them (Smith et al., 1999 ${ }^{\mathrm{b}}$ ). Apart from this possible influence related to the framing of questions, we also investigated some personal characteristics of the patients, selected on the basis of previous knowledge of their possible influence on patients' preferences and WTP values.

The health economics literature suggests that income positively influences WTP values (Donaldson, 1999; Smith et al. 1999 ; Drummond et al., 2005). Hence patients were asked their household incomes.

The medical literature indicates that previous experience of home care promotes preference for home care compared to hospital care. A Cochrane Collaboration literature review by Shepperd and Iliffe compared home care to hospital care in randomized studies (Shepperd and Iliffe, 2001). They concluded that patients who had no a priori preferences (since they agreed to participate in a randomized trial) generally expressed higher satisfaction after experiencing home care compared to hospital care. Accordingly, we asked our patients whether they had previously experienced home care, either for BT or any other procedure.

In the pilot study, patients were asked which BT management they preferred, and why. More precisely, they were proposed a list of possible reasons for their choice and were asked to state the importance of each reason using a 5-point Likert scale ranging from "not important at all" to "very important". Reasons were statistically compared according to importance using a Friedman's test, and a follow-up multiple comparison test. On the one hand, the most important reasons for preferring home BT were that it avoided disruption in daily life and that 
it spared transportation and waiting time in the hospital. On the other hand, hospital BT was mainly preferred because it allowed to clearly separate daily life from health care and because it was perceived as safer.

We assumed that, all other things being equal, patients would be all the more willing to spare transportation and waiting time as they lived far from the hospital and as their quality of life (including tiredness) was low. We thus selected two possible determinants of patients' preferences: distance between home and hospital, and a health-related quality of life index including tiredness as measured by the Functional Assessment of Cancer Treatment General scale (FACT-G) (Cella, 1993).

Considering safety, we assumed that patients would be all the more sensitive to this concern as their life was threatened, which was taken into account by collecting information on their disease stage. Three stage levels were used: curative, palliative and terminal.

Regarding daily life, patients' preferences referred to contradictory reasons. Some wished to avoid disruptions in daily activities, whereas others wanted to clearly separate daily life from health care. We collected data on patients' characteristics which could influence their feelings about daily life, i.e. marital status and having children at home or not, without foreknowledge of their influence on preferences. Other variables like standard demographics, gender, age and employment status were collected as controls, with no a priori assumption whatsoever on their possible influences.

\subsection{Sample characteristics}

On the study period, all 153 eligible patients consented to participate. However, 14 people could not be interviewed within the 48 hours following their BT either because of lack of 
availability or because they felt too tired. As a result, 139 patients were enrolled, which corresponds to a $90 \%$ response rate. Their characteristics are presented in Table I.

All patients' characteristics were documented by the patients themselves, except stage of disease which was assessed as curative, palliative or terminal by the oncologist in charge of the Home Care Unit at the Cancer Centre. The quality of life index measured using the FACT-G scale varied from 0 (worst possible situation) to 108 (best possible situation).

[Insert Table I over here]

Median monthly household income was between $1500 €$ and $2300 €$. Almost one in two respondents had previous experience of home BT on the one hand, and of home care (except home BT) on the other (43.2\% and $45.3 \%$ respectively). Distance from home to hospital was close to $35 \mathrm{~km}$ in average, with a high $100 \%$ variation coefficient. Quality of life according to the FACT-G scale was rather poor, with an average index of 61.7 (range 0-108). Regarding stage of disease, patients were almost equally distributed between curative stage on the one hand and palliative or terminal stage on the other.

Standard demographics were as follows: patients were 57.5 year old in average $(\mathrm{SD}=12.8)$ and $3.2 \%$ had a professional occupation. Finally, patients were equally distributed between males and females. 


\section{HEALTH ECONOMICS LITERATURE SURVEY: ECONOMETRIC MODELS FOR} ANALYZING DETERMINANTS OF WTP VALUES RESULTING FROM A BIDDING PROCESS

For Donaldson et al. (1998), the appropriate technique for econometric analysis of WTP data depends firstly on the type of question asked. For example, closed-ended questions and dichotomous choice with follow-up valuation only generate qualitative responses for WTP. This is why it is advised to use discrete choice models such as binary logit and probit for regression analysis (Johannesson et al., 1991; Johannesson et al., 1993). For data elicited by using a payment scale, the most appropriate econometric methodology is grouped data regression, also called interval regression or ordered logit/probit (Donaldson et al., 1998; Yasunaga et al., 2006; Bärnighausen et al., 2007). When using an open-ended question or a bidding process - as was the case in our study - the WTP values obtained are quantitative and several modeling methods have been proposed in the literature.

For responses obtained through the bidding process, the first regression analysis studies conducted in the health care field began in the 1980s and mostly estimated standard linear models by ordinary least squares (OLS) (Berwick and Weinstein, 1985; Thompson, 1986; O' Brien and Viramontes, 1994; Miedzybrobzka et al., 1995). ${ }^{1}$ However, the observed data for WTP responses are generally censored. When analyzing the distribution of WTP, we generally observe that the WTP variable does not take values below zero and has positive density at zero. The large proportion of zeros calls into question the continuity of the dependent variable and consequently the use of the classical multiple regression model. In the presence of data censoring, OLS estimation yields biased and inconsistent estimates because

\footnotetext{
The dependent variable of WTP values could be expressed in level, in logarithm or in square roots. Besides, Amin and Khondoker (2004) used a linear specification for WTP values, but supposed that they followed a log-normal distribution. Consequently, their estimations were based on maximum likelihood estimators instead of OLS.
} 
it fails to account for the qualitative difference between limit (zero) observations and nonlimit (continuous) observations. Consequently, all conclusions on the determinants of WTP are potentially erroneous. The Tobit model is the correct alternative frequently proposed for such censored data in contingent valuation literature in health economics (Donaldson et al., 1995; Kurth et al., 2004).

However, the underlying assumption in the Tobit model is that the same specification is used both for the continuous and the zero decision processes. This implies that the Tobit specification is relevant only if all zero realizations represent an economic decision, i.e. a real zero preference for the health care program under evaluation. Other quite reasonable determinants of zero observations, such as the presence of protest zeros which are common in contingent valuation surveys, also exist. Protest responses do not reflect individual patient preferences and have no economic significance (Lindsey, 1994; Jorgensen et al., 1999). They may reflect participants' objection to being asked to complete the questionnaires, or indicate an ideological position about the inappropriateness of placing monetary values on health. Protest patients refuse to reveal their real willingness even though it is positive. On the contrary, respondents genuinely place zero value on the WTP question, through stating that they have no strong (nil or very small) preference for it or they cannot afford to pay. Their responses have true economic significance. The issue at stake in the literature has thus been the development of appropriate empirical models to treat protest responses in addition to censored data. Several approaches have been proposed.

The simplest and most frequently used solution is to discard protest zeros. Nevertheless, this approach is generally incorrect from a statistical point of view: a sample selection bias is introduced if the socioeconomics and other personal characteristics of the protest patients are 
significantly different from the rest of the sample. Under such conditions, discarding protest responses produces biased estimates, which are not interpretable and unusable to test validity. The second solution is to indirectly address the issue of protest responses. Because zero responses may have explanations other than a genuine zero WTP, Donaldson et al. (1998) considered that positive WTP values and zero values could significantly differ in their determinants. In fact, positive WTP values stem from an economic decision-making process whereas zero values are a mixture of significant economic responses and protest responses. They proposed a specification of the censoring mechanism more flexible than Tobit, called the type II Tobit model (Amemiya, 1984) ${ }^{2}$ This model permits the coexistence of different patterns for the question of how much and whether to pay for the care under evaluation. One set of parameters determines the impact of the characteristics on the probability to record a positive WTP value, and the second set characterizes the determinants of the positive WTP amount:

$y_{i 1}= \begin{cases}1 \text { if } y_{i 1}^{*}=X_{i 1} \beta_{1}+u_{i 1}>0 & \text { (positive WTP value) } \\ 0 \text { else } & \text { (zero value for WTP) },\end{cases}$

and $y_{i}=\left\{\begin{array}{l}y_{i}^{*} \text { if } y_{i 1}=1 \\ 0 \text { else }\end{array}\right.$

with $y_{i}^{*}=X_{i 2} \beta_{2}+u_{i 2}, y_{i}$ the WTP value and $y_{i 1}^{*}$ and $y_{i}^{*}$ latent variables.

However, this approach does not differentiate zeros generated by economic decisions (genuine zero values) from zeros generated by non-economic decisions (protest zeros), which makes interpretation difficult.

This econometric specification is also called the sample selection model. The two-part model is a special case of the type II Tobit model. 
The third econometric approach, the double-hurdle model used by Dalmau-Matarrodona (2001), represents an interesting modification of the type II Tobit model by explicitly emphasizing protest responses. ${ }^{3}$ It decomposes the behaviour of individuals in the decisionmaking process in two parts: first, the reasons for deciding to participate or not in the contingent market offered, i.e. to give or not a protest response (participation equation: first hurdle), and second, the decision on the amount to consume, that is how much they are willing to pay for the procedure (consumption equation: second hurdle).

Participation equation:

$P_{i}=\left\{\begin{array}{l}1 \text { if } P_{i}^{*}=Z_{i} \gamma+u_{i}>0 \quad \text { (protest responses) } \\ 0 \text { else }\end{array}\right.$

Consumption equation:

$y_{i}=\left\{\begin{array}{l}y_{i}^{*} \text { if } y_{i}^{*}>0 \text { and } P_{i}=1 \\ 0 \text { if } P_{i}=0 \text { or if } P_{i}=1 \text { and } y_{i}^{*}=0,\end{array}\right.$

with $y_{i}^{*}=X_{i} \beta+u_{i}$

Compared with the type II Tobit model, the main advantage of the double-hurdle model is that the participation equation allows not only to determine the socioeconomic and personal characteristics of the protest respondents, but also to better define the WTP equation. In fact, in the type II Tobit model, the expression of the WTP equation depends on a selection equation which determines whether a positive WTP value is recorded. In the double-hurdle model, the WTP equation depends on the participation equation. But, because opposing protest responses to responses that reflect true preferences has more economic sense than opposing zero values to positive values, the selection is better taken into account in the

3 For a general presentation of this model, see Jones (1989, 1992), Blundell et al. (1987). 
double-hurdle methodology. Consequently, the consumption equation in the double-hurdle model allows to obtain unbiased and efficient estimations for the determinants of WTP for the whole sample. ${ }^{4}$ This is why the double-hurdle model seems to constitute the most robust approach for empirically testing validity in contingent valuation in the presence of censored data and protest responses. ${ }^{5}$

Even if the double-hurdle approach is attractive, it cannot provide reliable estimates when the number of protest responses is too small. In that case, the most appropriate econometric methodology is a truncated regression model (Mahmud, 2006). The estimation is based on strict positive WTP only and takes into account the elimination of all zero values to obtain valid results for all the patients: $y_{i}=y_{i}^{*}$ if $y_{i}>0$ with $y_{i}^{*}=X_{i} \beta+u_{i}$.

Parameters are estimated using the maximum likelihood principle rather than OLS. Indeed, with only limited numbers of protest responses, the type II Tobit model could be estimated, but the interpretation of the determinants of zero responses would remain problematic because the model does not differentiate genuine zero values from protest zeros. In this case, it is preferable to estimate a truncated regression model because all parameters have an economic interpretation.

\footnotetext{
Moreover, Flood and Gräsjo (1998) have shown, using Monte-Carlo simulations, that the double-hurdle specification is more general than the type II Tobit model. If the data are generated by type II tobit, the double-hurdle model still produces correct results, and if the data generation process is double-hurdle, serious biases can be avoided using double-hurdle instead of type II tobit.

5 The double-hurdle model can be viewed as a tobit method with selectivity.
} 


\section{ANALYZING THE DETERMINANTS OF OUR WTP VALUES}

\subsection{Choice of modeling}

As reported in Section 2, WTP data were collected in 139 patients. Forty-two patients responded that they would prefer to receive BT in the hospital versus 97 at home, i.e. $30 \%$ versus $70 \%$, respectively. Given that a large majority of patients preferred home BT to hospital BT, we decided to favour WTP data associated with preference for home BT. Accordingly, the 42 patients preferring hospital BT were considered to have real zero or negative WTP values for home BT.

Of the 97 patients preferring home BT, 3 expressed comprehension problems with the WTP question and their responses were eliminated from empirical analysis. Of the 94 remaining patients, 74 provided a strict positive WTP response and 20 chose a zero value indicating either protest responses or real zeros. The identification of protest zeros was based on responses to a follow-up question on the reasons for unwillingness to answer. Eight respondents reacted negatively to the payment vehicle and gave reasons for their zero WTP value such as "I have paid health insurance premiums all my life and I should not have to pay anything more", "the goal of this kind of study is that we pay more and more for care", "I do not want to pay for blood when the donors are voluntary". These 8 respondents were considered protest bidders. The other 12 patients who stated a zero response without any justification or because of low income were considered as real zeros. Apart from the 3 unusable and the 8 protest WTP responses, the mean WTP for home BT (as compared to hospital BT) among the 86 remaining patients was approximately $60 €(60.9 €)$ with a standard deviation close to $55 €(54.8 €)$. 
Censored data should receive a particular attention in our empirical analysis because, with the 42 patients who expressed a preference for hospital BT and were treated as real zero or negative WTP values and the 20 patients who preferred home BT but also stated a zero value ( 8 protest and 12 non protest responses), they represented $45 \%$ of the whole sample $(62 / 139)$. Since only 8 protest bidders were identified, it was not reasonable to estimate the participation equation of a double-hurdle model, i.e. to identify the variables influencing the choice of a respondent to reveal or not its real preferences. Nevertheless, discarding protest responses, even in limited number, could produce biased results. In this case, and as discussed above, the most appropriate econometric methodology is a truncated regression model. This model specifies the probability to observe the amount of WTP, conditional on the expression of a positive willingness to pay. The dependent variable $y_{i}$ was defined as the logarithm of the expressed WTP value in the model equation:

$$
y_{i}=y_{i}^{*} \text { if } y_{i}>0 \text { with } y_{i}^{*}=X_{i} \beta+u_{i}
$$

where $y_{i}$ is the value of the ith observation on the dependent variable $\log (\mathrm{WTP})$ and $y_{i}^{*}$ its corresponding latent value, described by the relation $y_{i}^{*}=X_{i} \beta+u_{i}$ with $X_{i}$ the independent variables that influence the WTP amount, $\beta$ the unknown parameters and $u_{i}$ the error term such as $u_{i} \rightarrow N\left(0, s^{2}\right)$. The likelihood function can be written as follows:

$$
L=\prod_{+} P\left(y_{i}=y_{i}^{*} \mid y_{i}>0\right)=\prod_{+} \frac{\frac{1}{s} f\left(\frac{y_{i}-X_{i} \beta}{s}\right)}{F\left(\frac{X_{i} \beta}{s}\right)}
$$

where $\prod_{+}$refers to the product over positive observations for $y_{i}$ and $F($.$) and f($.$) refers to the$ standard normal cumulative distribution function and density function, respectively. The unknown parameters are the solution to the maximization of the likelihood function. 


\subsection{Results}

The results of the truncated model are reported in Table II.

\section{[Insert Table II over here]}

According to the truncated regression model, several patient characteristics were significantly associated with the WTP value for home BT (as compared to hospital BT): income, experience of home care, distance from home to hospital, stage of disease, marital status (living with a partner) and professional occupation $(\mathrm{p}<0.05)$, as well as health-related quality of life $($ FACT-G) and age $(\mathrm{p}<0.1)$.

Initial bid was also significantly associated with WTP $(\mathrm{p}<0.01)$.

However, neither having children at home, previous experience of home BT, nor gender seemed to influence WTP values.

Compared with econometric results, none of the expected influences of pre selected possible determinants was invalidated (Table III).

[Insert Table III over here]

Considering patients characteristics, the WTP value increased with higher income, as commonly reported in the health economics literature on contingent valuation. Even if the WTP value did not seem to depend on previous experience of BT at home, it did depend on experience of home care other than BT. As said before, this is consistent with non-economic 
literature findings according to which previous experience of home care favours further preference for home care compared to hospital care.

Regarding the possible determinants selected on the basis of the results of our pilot study, we observed, as expected, that patients living far from the hospital stated a higher WTP for home BT (compared to hospital BT) than the others. Likewise, those with a higher health-related quality of life stated a lower WTP for home BT, which means that home BT was all the more appreciated, as compared to hospital BT, as patient quality of life (including tiredness) was low. This is in agreement with the results collected in the pilot study, which identified sparing painful transportation and waiting time as strong reasons for preferring home BT.

WTP for home BT was higher at early stages of the disease (curative) than at advanced stages, i.e. palliative or terminal. Referring to the safety concern expressed in the pilot study by patients preferring hospital BT, receiving hospital BT instead of home BT was all the more appreciated as patients' lives were threatened.

We could not hypothesize on the possible influences of other patients characteristics on WTP values. They were thus introduced in the model as control variables. Econometric results did not show any influence of either gender or having children at home. However, lower WTP values for home BT were obtained for patients living with a partner, which means that these appreciated hospital BT (compared to home BT) more than the others. This could result from a wish of clearly separating daily life from healthcare, as stated in the pilot study by patients. All other things being equal, WTP for home BT was higher for older people, and it was lower in patients with a professional occupation. These findings are not counterintuitive but could not be anticipated from what was known at the beginning of study.

Finally, the anchoring bias hypothesis was confirmed: all other things being equal, the WTP 
value for home BT increased when the initial bid was higher, which is consistent with results published in the health economics literature.

\section{DISCUSSION/CONCLUSION}

Home care may be an interesting alternative to hospital care, especially in chronic diseases. Accordingly, it is more and more used for cancer care, particularly for severely ill patients, including those at a palliative or terminal stage of their disease (Francks et al., 2000; Emanuel, 1996 ; Zimmerman et al., 2008). Home care has been extensively studied as a possible substitute for hospital care, regarding either costs or patients' quality of life and satisfaction (Brumley et al., 2007; Shepperd and Illife, 2001).

To our knowledge, the CV method had never been used for comparing home care to hospital care. However, it is an attractive approach for evaluating different types of health care management that differ only in patients' well-being during the treatment process and not in health outcomes (Ryan and Shackley, 1995; Olsen and Smith, 2001).

We considered the empirical situation of BT in cancer patients and we collected WTP values among BT users. In so doing, we chose to elicit use value, and not externality or option values. Indeed, we wanted to know whether a WTP questioning would be acceptable to severely ill patients, i.e. whether they would be willing not only to participate in the survey, but also to give non protest responses.

Protest responses could result from emotional and ethical concerns, as well as from social responsibility considerations (Jorgensen et al., 2000; Sayman and Onçüller, 2005; Meyerhoff et al. 2006), all dimensions which were encountered in our situation. Emotional and ethical aspects could be particularly important for patients at palliative or terminal stages; some of them even claimed that "health is invaluable". Social responsibility could also be an 
important concern since a survey using a WTP approach could call into question the principle of solidarity under which the cost of cancer care is completely covered by national health insurance in France.

The response rate of our study was high (90\%) and most patients $(70 \%)$ expressed a preference for home BT. Of the 94 patients who preferred home BT and who did not express understanding problems, only 8 gave protest responses, which corresponds to a less than $10 \%$ protest responses rate. Overall acceptability was thus high among BT users.

Our main objective was to test the validity of the CV method, namely its ability to elicit true preferences. For so doing, we chose a two-step approach. First, we identified possible determinants of our WTP values and their expected influences, on the basis of both economic and non economic literature. We also extrapolated from the findings of a previous pilot study. Second, we compared them to predicted influences as resulting from appropriate econometric analysis. The choice of appropriate econometrics for analyzing WTP values resulting from a bidding process is essential for obtaining relevant results. One must take into account that censored data at zero value correspond either to real zero values or to protest responses. As a consequence, suitable modeling should (1) involve the whole sample (including non zero responses, protest zeros and real zeros), (2) take the different behaviors at work into account (protest versus non protest responses). Based on a health economics literature survey, we concluded that the double-hurdle model would be the most appropriate approach to fulfill these two conditions. However, the estimation of the separation equation requires a sufficient number of protest responses. Because we had only few protest responses, we used a truncated regression model rather than a type II Tobit model in which estimated parameters for zero values would not be interpretable. 
None of the 7 hypothesized influences was invalidated by our econometric results. First, the anchoring bias hypothesis was confirmed. Regarding the possible influence of patients' characteristics, the WTP for home BT compared to hospital BT increased with the household income, with previous experience of home care, with living far from the hospital and with low quality of life. Conversely, the WTP for home BT was lower for advanced-stage (palliative or terminal) patients than for early-stage (curative) patients.

These results first underline the interest of using all a priori relevant sources of information for selecting possible determinants of WTP values. Second, because of the good consistency between expected and predicted influences of patients' characteristics on WTP values, our results may have some implications for policy-making. They suggest that, all other things being equal, home care should be considered in priority for people living far from hospital and people with low health-related quality of life. Regarding the stage of the disease, preference for home seemed to be lower among patients receiving palliative or terminal care than among those receiving curative care. This result may seem to contradict other medical literature findings according to which home care could improve satisfaction or quality of life in palliative patients (Zimmermann et al., 2008; Shepperd and Illife, 2001). However, we believe that there is no contradiction between the different conclusions since these authors did not compare patients' opinions across the different stages of disease.

From our study we conclude that, based on WTP values, the CV approach is acceptable to severely ill patients. Moreover, WTP values even demonstrate good validity, given that predicted determinants are consistent with expected ones. 
This study focuses on the interest of analyzing WTP values for documenting validity issues in the CV approach, but it might be important to investigate also the potential role of WTA. For a number of reasons, the use of WTA for decision making is not recommended. Studies investigating given health care programs have shown that WTA is generally higher and more scattered than WTP (Smith et al., 1999 ; Brown, 2005; Sayman and Onçüler, 2005; Whynes and Sach, 2007). On the basis of the WTA values also collected in our CV survey, we aim to investigate, in a further analysis, the potential role of WTA for testing CV validity. 


\section{ACKNOWLEDGEMENTS}

Special thanks go to Dr Yves Devaux, without whom this study would not have been possible. Yves Devaux is head of the oncology home care-coordination unit located at the comprehensive cancer center of Lyon.

We are also grateful to Valerie Kante, Josette Chalencon and the home care coordination team for their help in patient recruitment, to Marie-Dominique Reynaud for editorial assistance, and to Izabela Jelovac for valuable comments. 


\section{REFERENCES}

Amemiya T. 1984. Tobit models: a survey. Journal of Econometrics 24: 3-61.

Amin M, Khondoker F. 2004. A contingent valuation study to estimate the parental willingness-to-pay for childhood diarrhoea and gender bias among rural households in India. Health Research Policy and Systems 2: 3.

Bärnighausen T, Liu Y, Zhang X, Sauerborn R. 2007. Willingness to pay for social health insurance among informal sector workers in Wuhan, China: a contingent valuation study. BMC Health Services Research 7: 114.

Berwick D, Weinstein M. 1985 What do patients value? Willingness to pay for ultrasound in normal pregnancy. Medical Care 23: 881-893.

Blundell R, Ham J, Meghir C. 1987. Unemployment and female labour supply. Economic Journal 97: 44-64.

Brumley R, Enguidanos S, Jamison P, Seitz R et al. 2007. Increased Satisfaction with Care and Lower Costs: Results of a Randomized Trial of In-Home Palliative Care. Journal of the American Geriatrics Society 55(7): 993-1000.

Cella DF, Tulsky DS, Gray G et al. 1993. The Functional Assessment of Cancer Therapy Scale: development and validation of the general measure. Journal of Clinical Oncology 11(3): $570-579$.

Brown TC. 2005. Loss aversion without the endowment effect, and other explanations for the WTA-WTP disparity. J. of Economic Behavior \& Org. 57: 367-379

Dalmau-Matarrodona E. 2001. Alternative Approaches to obtain optimal bid values in contingent valuation studies and to model protest zeros. Estimating the determinants of individual's willingness to pay for home care services in day case surgery. Health Economics 16: 101-118. 
Diener A, O'Brien B, Gafni A. 1998. Health care contingent valuation studies: A review and classification of the literature. Health Economics 7: 313-326.

Donaldson C. 1999. Valuing the benefits of publicly-provided health care : Does 'ability to pay' preclude the use of'willingness to pay. Social Science and Medicine 49: 551-563.

Donaldson C, Jones A, Mapp T, Olson JA. 1998. Limited dependent variables in willingness to pay studies: applications in health care. Applied Economics 30: 667-677.

Donaldson C, Shackley P, Abdalla M, Miedzybrodzka Z. 1995. Willingness to pay for antenatal carrier screening for cystic fibrosis. Health Economics 4: 439-452.

Drummond MF, Sculpher MJ, Torrance GW, O’Brien B, Stoddart GL. 2005. Methods for the economic evaluation of health care programmes. Third Edition. Oxford, Oxford University press.

Emanuel EJ. 1996. Cost saving at the end of life. What do the data show? JAMA 275:19071914.

Flachaire E, Hollard G. 2007. Starting point bias and respondent's uncertainty. Ressource and Energy Economics 29: 183-194.

Flood L, Gräsjo U. 1998. Regression Analysis and Time Use Data: A Comparison of Microeconometric Approaches with Data from the Swedish Time Use Survey (HUS). Working Papers in Economics no. 5. School of Economics and Commercial Law, Göteborg University, Sweden.

Franks PJ, Salisbury C, Bosanquet N, Wilkinson EK et al. 2000. The level of need for palliative care: a systematic review of the literature. Palliat Med 14(2): 93-104.

Idri S, Catoni MP, Si Ali H, Patte R, Briere J, Baudelot J, Courtois F. 1996. La transfusion à domicile ? Une alternative à l'hôpital de jour. Transfusion Clinique et Biologique 3: 235-239. Johannesson M, Jonsson B, Borgquist L. 1991. Willingness to pay for antihypertensive therapy- results of a Swedish pilot study. Journal of Health Economics 10: 461-474. 
Johannesson M., Johansson PO, Kristrom B., Gerdtham UG. 1993. Willingness to pay for antihypertensive therapy -further results. Journal of Health Economics 12: 95-108.

Jones AM. 1989. A double-hurdle model of cigarette consumption. Journal of Applied Econometrics 4: 23-39.

Jones AM. 1992. A Note on Computation of the Double Hurdle Model with Dependence with An Application to Tobacco Expenditure. Bulletin of Economic Research 44: 67-73.

Jorgensen BS, Syme GJ, Bishop, BJ, Nancarrow BE. 1999. Protest Responses in Contingent Valuation. Environmental and Resource Economics 14: 131-150.

Jorgensen, BS, Wilson, MA, Heberlein TA. 2000. Fairness in the Contingent Valuation of Environmental Integrated knowledge for ecological economics: a database to support ecosystem services evaluation. Ecological Economics 36: 133-148.

Kahneman D, Tversky A.1979. Prospect Theory: An Analysis of Decision Under risk. Econometrica 47: 263-291.

Kurth A, Waever M, Lodhart D, Bielinski L. 2004. The Benefit of Health Insurance Coverage of Contraceptives in a Population-Based Sample. American Journal of Public Health 94: $1330-1332$.

Lindsey G. 1994. Market models, protest bids, and outliers in contingent valuation. Journal of Water Resources Planning and Management 120: 121-129.

Madgwick KY, Yardumian A. 1999. A home blood transfusion programme for betathalassaemia patients. Transfusion Medicine 9: 135-138.

Mahmud M. 2006. Contingent Valuation of Mortality Risk Reduction in Developing Countries: A Mission Impossible? Keele Economics Research Papers 1: 28.

Meyerhoff J, Liebe U. 2006. Protest Beliefs in Contingent Valuation: Explaining Their Motivation. Ecological Economics 57 (3): 583-594. 
Miedzybrodzka Z, Semper J, Shackley P, Abdalla M, Donaldson C. 1995. Stepwise or couple antenatal carrier screening for cystic fibrosis? Women's preferences and willingness to pay, Journal of Medical Genetics 32: 282-283.

Mitchell RC, Carson RT. 1989. Using Surveys to Value Public Goods: The Contingent Valuation Method. Baltimore: John Hopkins University Press.

National Oceanic and Atmospheric Administration (NOAA). 1994. Natural resource damage assessments: proposed rules. Federal Register 59: 1062-1191.

O'Brien B, Viramontes JL. 1994. Willingness to pay: a valid and reliable measure of health state preference? Medical Decision Making 14: 289-297.

O’Brien B, Gafni A. 1996. When do the "dollars" make sense?. Medical Decision Making 16: 288-299.

Olsen J.A, Smith R. 2001. Theory versus practice: A review of willingness to pay in health and health care. Health Economics 10: 39-52.

Ryan M, Shackley P. 1995. Assessing the benefits of health care: how far should we go? Quality in Health Care 4: 207-213.

Sayman S, Onculer A. 2005. Effects of study design characteristics on the WTA-WTP disparity: A meta analytical framework. Journal of Economic Psychology 26: 289-312.

Shepperd S, Iliffe S. 2001. Hospital at home versus in-patient hospital care (Cochrane Review). In: The Cochrane Library Issue 1, Chichester, UK: John Wiley \& Sons.

Smith R, Olsen JA, Harris A. $1999^{\mathrm{a}}$. A review of methodological issues in the conduct of willingness to pay studies in health care II: administration of CV survey. Centre for Heath Program Evaluation, Monash University, working paper 85.

Smith R, Olsen JA, Harris A. $1999^{\mathrm{b}}$. A review of methodological issues in the conduct of willingness to pay studies in health care III: issues in the analysis and interpretation of WTP data. Centre for Heath Program Evaluation, Monash University, working paper 86. 
Smith RD. 2003. Construction of the contingent valuation market in health care: a critical assessment. Health Economics 12: 609-628.

Smith RD. 2006. It's Not Just What You Do, It's the Way That You Do It: The Effect of Different Payment Card Formats and Survey Administration on Willingness to Pay for Health Gain. Health-Economics 15: 281-293.

Smith RD. $2007^{\text {a }}$. The role of 'reference goods' in contingent valuation: should we help respondents to 'construct' their willingness to pay ? Health Economics 16: 1319-1332.

Smith RD. $2007^{\mathrm{b}}$. Use, option and externality values: are contingent valuation studies in health care mis-specified? Health Economics 16: 861-869.

Thompson MS. 1986. Willingness to pay and accept risks to cure chronic disease. American Journal of Public Health 76: 392-396.

Yasunaga H, Ide H, Imamura T, Ohe K. 2006. Willingness to pay for health care services in common cold, retinal detachment and myocardiac information: an internet survey in Japon. BMC Health Services Research 6: 12.

Yeung RY, Smith RD, Ho LM, Johnston JM, Leung GM. 2006. Empirical implications of response acquiescence in discrete-choice contingent valuation. Health Economics 15:10771089.

Zimmermann C, Riechelmann R, Krzyzanowska M, Rodin G, Tannonck I. 2008. Effectiveness of specialized care : A systematic review. JAMA 299:1698-1709. 


\section{TABLES}

\section{Table I. Patient characteristics}

\begin{tabular}{|c|c|}
\hline Characteristics & Mean \pm SD or number of patients $(\%)$ \\
\hline \multicolumn{2}{|l|}{ Income $^{(1)}$} \\
\hline$<800 €$ & $16(12.2 \%)$ \\
\hline $800-1499 €$ & $37(28.2 \%)$ \\
\hline $1500-2299 €$ & $34(25.9 \%)$ \\
\hline $2300-2999 €$ & $22(16.8 \%)$ \\
\hline$\geq 3000 €$ & $22(16.8 \%)$ \\
\hline Experience of home BT (yes/no) & $60(43.2 \%) / 79(56.8 \%)$ \\
\hline Experience of home care (except home BT)(yes/no) & $63(45.3 \%) / 76(54.7 \%)$ \\
\hline Distance from home to hospital $(\mathrm{km})($ mean $\pm \mathrm{SD})$ & $34.9 \pm 40.6$ \\
\hline FACT-G ${ }^{(2)}$ & $61.7 \pm 13.9$ \\
\hline
\end{tabular}

Stage of disease

Curative $70(50.4 \%)$

Palliative $55(39.6 \%)$

Terminal $14(10.1 \%)$

Living with a partner (yes/no) $102(73.4 \%) / 37(26.6 \%)$

Children at home (yes/no) $40(28.8 \%) / 99(71.2 \%)$

Male/female $69(49.6 \%) / 70(50.4 \%)$

Age (years) $57.5 \pm 12.8$

Professional occupation (yes/no) $60(43.2 \%) / 79(56.8 \%)$

\footnotetext{
(1) Net monthly household income before income tax (8 missing data)

${ }^{(2)}$ Functional Assessment of Cancer Care General scale (6 missing data)
} 
Table II. Results of the truncated regression model of WTP (log) values

\begin{tabular}{|c|c|c|c|}
\hline Explanatory variables & Coefficient & $\mathrm{SE}$ & $p$-value \\
\hline Intercept & 3.17630 & 0.514 & $0.000^{* * * *}$ \\
\hline Income $(1=2300 €$ or more $)$ & 1.02655 & 0.142 & $0.000^{* * *}$ \\
\hline Experience of home BT (1=yes) & 0.16635 & 0.160 & 0.298 \\
\hline Experience of home care, except BT ( $1=$ yes $)$ & 0.41905 & 0.124 & $0.001^{* * *}$ \\
\hline Distance to hospital (continuous scale) & 0.00365 & 0.002 & $0.045^{* *}$ \\
\hline FACT-G (continuous scale) & -0.00944 & 0.005 & $0.060^{*}$ \\
\hline Stage of disease ( $1=$ curative; $0=$ palliative or terminal $)$ & 0.30030 & 0.128 & $0.019^{* *}$ \\
\hline Living with a partner $(1=y e s)$ & -0.45585 & 0.162 & $0.005^{* * *}$ \\
\hline Children at home $(1=$ yes $)$ & 0.21794 & 0.133 & 0.102 \\
\hline Gender $(1=$ male $)$ & 0.17425 & 0.130 & 0.180 \\
\hline Age (continuous scale) & 0.01247 & 0.006 & $0.053^{*}$ \\
\hline Professional occupation ( $1=$ yes) & -0.49574 & 0.146 & $0.001^{* * *}$ \\
\hline Initial Bid $(1=76 € ; 0=38 €)$ & 0.37818 & 0.125 & $0.003^{* * *}$ \\
\hline$\sigma$ & 0.46671 & 0.040 & $0.000^{* * *}$ \\
\hline Number of observations & 68 & & \\
\hline Log likelihood & -44.66932 & & \\
\hline
\end{tabular}


Table III. Expected and predicted influences of possible determinants of WTP (log) values

\begin{tabular}{|c|c|c|}
\hline & Expected & Predicted \\
\hline Explanatory variables & influence & influence \\
\hline Income $(1=2300 €$ or more $)$ & $+{ }^{\mathrm{a}}$ & $+* * *$ \\
\hline Experience of home BT (1=yes) & $+{ }^{b}$ & NS \\
\hline Experience of home care, except BT (1=yes) & $+{ }^{b}$ & $+* * *$ \\
\hline Distance to hospital (continuous scale) & $+{ }^{\mathrm{c}}$ & $+* *$ \\
\hline FACT-G (continuous scale) & $-{ }^{c}$ & $-*$ \\
\hline Stage of disease $(1=$ curative; $0=$ palliative or terminal $)$ & $+{ }^{\mathrm{c}}$ & $+* *$ \\
\hline Living with a partner $(1=$ yes $)$ & $?^{\mathrm{c}}$ & $-* * *$ \\
\hline Children at home $(1=$ yes $)$ & $?^{\mathrm{c}}$ & NS \\
\hline Gender $(1=$ male $)$ & $?$ & NS \\
\hline Age (continuous scale) & $?$ & $+*$ \\
\hline Professional occupation ( $1=$ yes $)$ & $?$ & $-* * *$ \\
\hline Initial Bid $(1=76 € ; 0=38 €)$ & $+{ }^{a}$ & $+* * *$ \\
\hline
\end{tabular}

